\title{
Usage of standards in order to integration of hospital information systems
}

\author{
Leila Shahmoradi1(D), Maryam Ebrahimi ${ }^{2}$, Somayeh Shahmoradi ${ }^{3}$, Ahmadreza Farzaneh \\ Nejad $^{4}$ (D), Hajar Moammaie ${ }^{5}$, Mahdi Habibi-Koolaee ${ }^{6^{*}(\mathbb{D})}$
}

\begin{abstract}
${ }^{1}$ Department of Health Information Management, Faculty of Allied Medical Sciences, Tehran University of Medical Sciences, Tehran, Iran ${ }^{2}$ Department of Health Information Technology, Neyshabur University of Medical Sciences, Neyshabur, Iran

${ }^{3} \mathrm{MSc}$, Department of Computer Engineering, Islamic Azad University, Tehran, Iran

${ }^{4}$ Department of Health Information Management, Faculty of Allied Medical Sciences, Tehran University of Medical Sciences, Tehran, Iran ${ }^{5} \mathrm{MSc}$ in MBA, Tehran University of Medical Sciences, Tehran, Iran

${ }^{6}$ Department of Education, Golestan University of Medical Sciences, Gorgan, Iran
\end{abstract}

\begin{tabular}{|c|c|}
\hline Artic & A B S T R A C T \\
\hline $\begin{array}{l}\text { Article type: } \\
\text { Research }\end{array}$ & \multirow{2}{*}{$\begin{array}{l}\text { Introduction: Data exchange across healthcare facilities is a major issue in } \\
\text { healthcare information systems. Standards play an important role in the } \\
\text { context of communication. In this paper, we surveyed the usage of } \\
\text { standards in the hospital information systems (HISs) in the affiliated } \\
\text { hospitals of Tehran University of Medical Sciences. }\end{array}$} \\
\hline $\begin{array}{l}\text { Article History: } \\
\text { Received: 2020-01-08 }\end{array}$ & \\
\hline $\begin{array}{l}\text { Accepted: 2020-03-08 } \\
\text { Published: 2020-03-08 }\end{array}$ & \multirow{2}{*}{$\begin{array}{l}\text { Material and Methods: This survey was performed in 2014-2015 } \\
\text { Seventeen hospitals that had an HIS were surveyed. The data were } \\
\text { collected using a structured questionnaire. The design of the questionnaire } \\
\text { was based on a literature review and consisted of three parts. Descriptive } \\
\text { statistics were used to analyze the data. }\end{array}$} \\
\hline $\begin{array}{l}\text { *Corresponding author: } \\
\text { Mahdi Habibi-Koolaee }\end{array}$ & \\
\hline Department of Education, Goles & \multirow{3}{*}{$\begin{array}{l}\text { Results: XML, HL7 and DICOM are commonly used international } \\
\text { interchange standards. In the case of security standards, } 76.5 \% \text { of HISs do } \\
\text { not support the HIPPA and CEN TC } 251 \text { security standards. ICD was the } \\
\text { most commonly used terminology standard in the HISs. Several studies } \\
\text { have indicated that HISs should cover data exchange, security and } \\
\text { terminology standards to provide integration of heterogeneous systems. }\end{array}$} \\
\hline $\begin{array}{l}\text { University of Medical Sciences, } \\
\text { Gorgan, Iran }\end{array}$ & \\
\hline $\begin{array}{r}\text { Keyword } \\
\text { Standa }\end{array}$ & \\
\hline $\begin{array}{l}\text { Standards } \\
\text { Information Systems } \\
\text { Hospital Information Systems } \\
\text { Iran }\end{array}$ & $\begin{array}{l}\text { Conclusion: In the current study, the role of standards in the architecture } \\
\text { of the HISs was inconspicuous. To make the HIS effective, it is necessary to } \\
\text { consider the standards when developing the system. In this matter, } \\
\text { legislation could help. }\end{array}$ \\
\hline
\end{tabular}

Cite this paper as:

Shahmoradi L, Ebrahimi M, Shahmoradi S, Farzaneh Nejad A, Moammaie H, Habibi-Koolaee M. Usage of Standards in Order to Integration of Hospital Information Systems. Front Health Inform. 2020; 9: 28. DOI: 10.30699/fhi.v9i1.215

\section{INTRODUCTION}

In recent years, there has been increasing interest in the use of information systems in the healthcare sector. Hospital information systems (HISs) are one of the most widely used systems. HIS is a computer system designed to simplify the management of a hospital's clinical and administrative information and to improve the quality of health care [1]].

For effective activities and services in hospitals, the HIS plays an important role to provide information for managers. Using information systems can help clinicians to enhance the quality of care [2] .

HISs consist of several parts, including computerized physician order entry (CPOE), laboratory information system (LIS), radiology information system (RIS), and etc. These parts need to exchange data. Hence, the integration of the subsystems of the
HIS is essential []] .

Complete integration should cover three aspects of integration, including data integration, function integration and workflow integration [4]. Hospitals have many advantages with data integration. Although, it is currently taking place in many hospitals, there are still many difficult issues, such as the low quality of patient data []․

The HIS should provide an integrated electronic medical record (EMR). Because of the heterogeneous HISs, patient information is distributed in different resources. To have an integrated EMR, it is important to integrate the HISs []].

Several attempts have been made to integrate heterogeneous information systems in the health care domain [4, 7-12]. For instance, the Common Object Request Broker Architecture (CORBA) that 
allows sharing of activities, such as access and communication between different systems. Standards, such as Health Level 7 (HL7), Integrated Healthcare Enterprise (IHE) and Digital Imaging and Communications in Medicine (DICOM), introduce and provide guidelines for the exchange of messages among different systems. This enables the integration of heterogeneous systems $[\underline{8}, \underline{13}]$.

The role of standardization has been identified as a major contributing factor for integration. Standardization is a vital issue during information exchange from one place to another, especially outside organizations [14].

Currently, the most common standards in the world (i.e., HL7, International Classification of Diseases (ICD) and DICOM) are used in the Islamic Republic of Iran. With regard to the development of SEPAS (Persian abbreviation for the national electronic health record system) and the necessity of the connectivity of the HISs from all hospitals in the country to this system, the standard reference model is defined as the ISO 13606 standard [ $\underline{15}]$. The ISO 13606 standard provides syntactic and semantic capabilities (through a dual model approach) as well as terminology, security and interface considerations for the standardized exchange of EMR [16].

This paper attempts to survey the usage of standards in the hospital information systems (HISs) in the affiliated hospitals of Tehran University of Medical Sciences.

\section{MATERIAL AND METHODS}

This was a descriptive study, performed in 20142015. All affiliated hospitals of Tehran University of Medical Sciences (TUMS) that had an HIS were included in the study. The initial sample consisted of seventeen hospitals of which some did not complete all interviews.

A structured questionnaire was used to collect data. The design of the questionnaire was based on a literature review of journal articles, books and other available library resources about integration tools and techniques such as guidelines and protocols. The questionnaire consisted of three parts. The first part dealt with demographic information about participants, including six closed questions, such as name, position and specialty of interview.

The second part of the questionnaire consisted of information about hospital information systems in terms of the name of the developer and supporter companies, the subsystems of the HIS and the relation among them with six closed and semi-open questions. Finally, the third part of the questionnaire dealt with the identification of standards types used to exchange information and integration of HISs with 19 questions (closed and open), for instance, international standards used to exchange data, security standards, and standards used by the subsystems of HIS.

Viewpoints of participants were gathered. Descriptive statistics was used to analyze the data. The participants were informed about the objectives of the study and were informed that the results of the study will be published by maintaining trusteeship.

\section{RESULTS}

Descriptive statistical analysis was used to measure the amount of the usage of standards in the seventeen hospitals affiliated to TUMS. The results are shown in the following tables.

Table 1 shows the participants' positions in the hospitals. Over half of participants was the head of HIS and Information Technology (IT) department.

Table 1: Frequency of the participants' position

\begin{tabular}{|l|c|c|}
\hline Position & Frequency & Percentage \\
\hline Head of HIS* department & 4 & 23.5 \\
\hline $\begin{array}{l}\text { Head of Informatics } \\
\text { department }\end{array}$ & 2 & 11.8 \\
\hline $\begin{array}{l}\text { Head of Information } \\
\text { Technology department }\end{array}$ & 5 & 29.3 \\
\hline Head of Production unit & 1 & 5.9 \\
\hline Head of Network unit & 2 & 11.8 \\
\hline Head of Software unit & 1 & 5.9 \\
\hline Not specified & 2 & 11.8 \\
\hline Total & 17 & 100 \\
\hline
\end{tabular}

Table 2 indicates that only $17.6 \%$ of HISs use the IEEE 11073 standard. Approximately three-quarters of HISs (82.4\%) did not cover the IEEE 11073 standards. The eXtensible Markup Language (XML) $(58.8 \%)$ was the most commonly used international data interchange markup language.

Table 2: Frequency of the international data interchange standards in hospital information systems

\begin{tabular}{|l|c|c|}
\hline $\begin{array}{l}\text { International data } \\
\text { interchange standards }\end{array}$ & $\begin{array}{l}\text { Frequency } \\
\text { (Out of 17) }\end{array}$ & Percentage \\
\hline IEEE $11073^{*}$ & 3 & 17.6 \\
\hline IHE $^{*}$ & 0 & 0 \\
\hline $\mathrm{X}^{*} \mathrm{~N}^{*}$ & 0 & 0 \\
\hline NCPDP $^{*}$ & 0 & 0 \\
\hline XML $^{*}$ & 10 & 58.8 \\
\hline DICOM $^{*}$ & 5 & 29.4 \\
\hline HL7 $^{*}$ & 6 & 35.3 \\
\hline CEN TC 251 & 2 & 11.8 \\
\hline Open EHR & 1 & 5.9 \\
\hline
\end{tabular}

*IEEE: Institute of Electrical and Electronics Engineers; IHE: Integrated Healthcare Enterprise; NCPDP: National Council for Prescription Drug Programs; XML: eXtensible Markup Language; DICOM: Digital Imaging and Communications in Medicine; HL7: Health Level 7; CEN TC 251: European Committee for Standardization technical committee 251; Open EHR: Open Electronic Health records 
Table 3 illustrates the HIS standards for information security and terminology in data exchange. Three HISs support the HIPAA security standards, and only one HIS supports the CEN TC251 security standards. The majority of HISs (76.5\%) do not support any security standards.

Table 3: Frequency of the security standards in hospital information systems

\begin{tabular}{|l|c|c|}
\hline Security standards & Frequency & Percentage \\
\hline $\begin{array}{l}\text { HIPAA }^{*} \text { security } \\
\text { standard }\end{array}$ & 3 & 17.6 \\
\hline CEN TC $251^{*}$ & 1 & 5.9 \\
\hline $\begin{array}{l}\text { Did not support the } \\
\text { security standards }\end{array}$ & 13 & 76.5 \\
\hline total & 17 & 100 \\
\hline
\end{tabular}

*HIPPA: Health Insurance Portability and Accountability Act; CEN TC 251: European Committee for Standardization technical committee 251

In table 4 , the main terminology standards for data integration are illustrated. The International Classification of Diseases (ICD) (70.6\%) is the most common terminology standard used in HISs. The result also shows that only one HIS uses RxNORM and LOINC. These two standards are needed for the integration of Pharmacy Information Systems (PIS) and Laboratory Information Systems (LIS), respectively.

Table 4: Frequency of the terminology standards in hospital information systems

\begin{tabular}{|l|c|c|}
\hline $\begin{array}{l}\text { Terminology } \\
\text { standards }\end{array}$ & $\begin{array}{l}\text { Frequency } \\
\text { (out of 17) }\end{array}$ & Percentage \\
\hline RxNORM & 1 & 5.9 \\
\hline LOINC $^{*}$ & 1 & 5.9 \\
\hline SNOMED-CT & 2 & 11.8 \\
\hline ICD $^{*}$ & 12 & 70.6 \\
\hline
\end{tabular}

*RxNORM: standardized nomenclature for clinical drugs; LOINC: Logical Observation Identifiers Names and Codes; SNOMED-CT: Systematized Nomenclature of Medicine--Clinical Terms; ICD: International Classification of Diseases

\section{DISCUSSION}

The present study was designed to determine the usage of standards in HISs. The amount of the use of IEEE standards, international data exchange standards, security standards and terminology standards were investigated. The most interesting finding was the high usage of ICD (terminology standard) in HISs.

Schmitt et al. suggested that to solve the problem of interoperability on the application level it is necessary that all devices have a common language to understand each other by means of a common nomenclature, data type, message syntax and encoding rules [17]. The ISO 11073/IEEE 1073 family of standards is an international standard that specifies the information model and communication protocol between medical devices and external information systems (i.e. HISs) [18] and enables medical devices to interoperate with bedside devices [19].

As the results show, only 3 out of 17 HIS use the IEEE 11073 standards. It is difficult to explain this result, but it might be related to the objective of HIS design and implementation. For example, most HISs in this study aimed to meet financial needs, and the interoperability between devices are not considered. However, the findings of this study are not consistent with the study of Vlach et al. which used standards, such as IEEE standards, for integration of a vital sign monitor devices with HIS and a central registry [11].

HL7 is one of the common international communication standards for data integration. For example, HL7 CDA (clinical document architecture) is a standard for the representation of clinical documents. HL7 CDA is based on XML and can facilitate information exchange between different systems [20]. According to the results of this study, XML, HL7 and DICOM are the most common data interchange standards of HISs. These findings of the current study are consistent with those of Xuchen Li et al. who used an XML based middleware to integrate clinical data and used HL7 v2.3.1 as the communication interface of the middleware [10]. The results are consistent with Heitmann et al. who used XML for standardization of the communication between hospital information systems and physician offices [21].

IHE is more than a standard [22]. The first focus of IHE was to define how to use HL7 and DICOM to resolve the communication of current information systems in radiology [23]. None of the IHE, X12N and NCPDP standards are used in the HISs. However, many studies indicated the important role of these standards to integrate heterogeneous systems. For example, in 2005, Xudong Lu et al. indicated that IHE can used for integration of information systems using a common framework and can also employ HL7, DICOM and IEEE 11073 to connect devices, terminals and HISs [ $\underline{4}$ ].

To maintain various patient data across several different systems in compliance with legislative requirements, HIPAA had specific requirements for patient records, especially when data interchange is needed [24]. In this study, $76.5 \%$ of HISs do not support HIPAA and CEN TC 251 security standards. This finding was unexpected. The importance of this issue is expressed in other studies [25, 26]. An explanation for this might be that the HIS designers are not familiar with security standards in the healthcare domain. It can thus be suggested that systems be legally required to consider the security standards in the development of any information system. Some studies have pointed to the security mechanisms in the hospitals of Iran. For example, Sharifian et al. found a lack of appropriate security 
technical and administrative infrastructure. They also stated that to increase the application of required administrative security safeguards, operational planning must be implemented [27].

Using standardized terminology is one of the integration tools for the healthcare system. International Classification of Diseases (ICD) and SNOMED CT are two common terminology standards [28]. ICD is used for external reporting requirements and also when data aggregation is required. Unlike the ICD, which is not designed for primary documentation of clinical data, the SNOMED $\mathrm{CT}$, a reference terminology, is designed for coding of clinical information during the course of patient care. It provides a common language that enables consistent sharing and aggregation of health care data among organizations [루].

In the current study, these two standards play an important role in the integration issues in HISs, but the use of LOINC and RxNORM to provide integration of subsystems of the HIS is not common. There are several possible explanations for this result. ICD and SNOMED CT are the accepted terminology for HISs from the Ministry of Health and Medical Education. However, only 70.6\% and 11.8\% of HISs use the ICD and SNOMED CT standards, respectively.

These findings suggest that the HIS developer company and hospital managers should consider the use of standards in information systems to enable integration in information exchange between heterogeneous systems. To increase efficiency of services, HISs can provide useful information for hospital managers to help clinicians to improve healthcare quality. Hence, HISs require standards to be useful.

Overall, these findings have important implications for understanding of the data integration status in HISs, which are currently in use in TUMS.

\section{CONCLUSION}

This paper has investigated the status of the integration of HISs in the affiliated hospitals of TUMS regarding the use of standards. The purpose was to answer the question of which standards are used in the HISs, international data interchange standards, security standards as well as terminology standards.

This study has found that most of the HISs did not support the above standards. Many of these systems require communication with each other and other devices, and these systems did not address the integration issues, especially at the data level.

Taken together, these results suggest that hospitals should consider the standardization needs for HISs. Without standards, they cannot compete and communicate with other organizations. Some of the intervention in the treatment period required communication between different systems. Inability of the data integration between these systems may affect the patient care process.

Further work needs to be performed to investigate the reason why the information systems do not follow the standards. Unless governmental bodies (i.e., Ministry of Health) mandate the use of standards, the integration of the HISs will not be completed.

\section{AUTHOR'S CONTRIBUTION}

All the authors approved the final version of the manuscript.

\section{CONFLICTS OF INTEREST}

The authors declare no conflicts of interest regarding the publication of this study.

\section{FINANCIAL DISCLOSURE}

This research was supported financially by the Tehran University of Medical Sciences (TUMS) Grant No. 93-03-31-26335.

\section{REFERENCES}

1. Degoulet P. Hospital information systems. In: Venot A, Burgun A, Quantin C [ed]. Medical Informatics, eHealth. Paris: Springer. 2014; 289-313.

2. Amiri M, Sadeghi E, Khosravi A, Chaman R. Selfassessment of managers and network operators about the effect of hospital information system on the performance and processes of Imam Hossein hospital in Shahroud. Health Information Management. 2011; 8(4): 490-9.

3. Karimi Hosseini N, Nordin JG, Mahdiani M, Sadrzadeh Rafiei S. A low cost hospital information system: A case study of the Mehr hospital in Mashhad city. Research Journal of Applied Sciences, Engineering and Technology 2014;7(7): 1132-8.

4. Lu X, Duan H, Li H, Zhao C, An J. The architecture of enterprise hospital information system. Conf Proc IEEE Eng Med Biol Soc. 2005;2005:6957-60. PMID: 17281875 DOI: $\quad 10.1109 /$ IEMBS.2005.1616106 [PubMed]

5. Cruz-Correia R, Vieira-Marques P, Ferreira A, Oliveira-Palhares E, Costa P, Costa-Pereira A. Monitoring the integration of hospital information systems: How it may ensure and improve the quality of data. Stud Health Technol Inform. 2006;121:17682. PMID: 17095815 [PubMed]

6. Trinkunas J, Tuinyliene E, Puronaite R. Research on hospital information systems integration to national electronic health record system. International 
Conference BIOMDLORE: IEEE; 2018.

7. Karampela I, Tzortzis E, Kefala I, Zygoura E, Mantzana V, Armaganidis A. Proposal for the implementation of quality standards in a medical unit through integration to the hospital information system. Stud Health Technol Inform. 2017; 238: 1858. PMID: 28679919 [PubMed]

8. Ko L-F, Lin J-C, Chen C-H, Chang J-S, Lai F, Hsu KP, et al. HL7 middleware framework for healthcare information system. International Conference on eHealth Networking, Applications and Services. New Delhi: IEEE; 2006.

9. Frascina AC. The integration of hospital information systems through user centerd design. [PhD Thesis] Sheffield: Sheffield Hallam University; 1994.

10. Li X, Vojisavljevic V, Fang Q. An XML based middleware for ECG format conversion. Conf Proc IEEE Eng Med Biol Soc. 2009; 2009: 1691-4. PMID: 19964551 DOI: $10.1109 /$ IEMBS.2009.5333907 [PubMed]

11. Vlach K, Jirka J, Cernohorsky J. Integration of DASH and ICS 3000 devices with hospital information system and REPACE central registry. Conf Proc IEEE Eng Med Biol Soc. 2012; 2012: 5798-801. PMID: 23367247 DOI: $10.1109 /$ EMBC.2012.6347312 [PubMed]

12. Strahle M, Ehlbeck M, Prapavat V, Kück K, Franz F, Meyer JU. Towards a service-oriented architecture for interconnecting medical devices and applications. Joint Workshop on High Confidence Medical Devices, Software, and Systems and Medical Device Plug-andPlay Interoperability. Boston: IEEE; 2007.

13. Sujansky WV, Overhage JM, Chang S, Frohlich J, Faus SA. The development of a highly constrained health level 7 implementation guide to facilitate electronic laboratory reporting to ambulatory electronic health record systems. J Am Med Inform Assoc. 2009 MayJun; 16(3): 285-90. PMID: 19261950 DOI: $10.1197 /$ jamia.M2610 [PubMed]

14. Modaresnezhad M, Vahdati A, Nemati H, Ardestani A, Sadri F. A rule-based semantic approach for data integration, standardization and dimensionality reduction utilizing the UMLS: Application to predicting bariatric surgery outcomes. Comput Biol Med. 2019; 106: 84-90. PMID: 30708220 DOI: 10.1016/j.compbiomed.2019.01.019 [PubMed]

15. Center of Statistics and Information Technology Management. SEPAS middleware concepts: Electronic health records development plan. Tehran: Ministry of Health and Medical Education; 2011.

16. Muñoz P, Trigo JD, Martínez I, Muñoz A, Escayola J, García J. The ISO/EN 13606 standard for the interoperable exchange of electronic health records. Journal of Healthcare Engineering. 2011; 2(1): 1-24.

17. Schmitt L, Falck T, Wartena F, Simons D. Novel ISO/IEEE 11073 standards for personal telehealth systems interoperability. Joint Workshop on High Confidence Medical Devices, Software, and Systems and Medical Device Plug-and-Play Interoperability. Boston: IEEE; 2007.

18. Soceanu A, Egner A, Moldoveanu F. Towards interoperability of eHealth system networked components. International Conference on Control Systems and Computer Science, Bucharest: IEEE; 2013.

19. Yao J, Warren S. Applying the ISO/IEEE 11073 standards to wearable home health monitoring systems. J Clin Monit Comput. 2005; 19(6): 427-36. PMID: 16437294 DOI: 10.1007/s10877-005-2033-7 [PubMed]

20. Muller ML, Uckert F, Burkle T, Prokosch HU. Crossinstitutional data exchange using the clinical document architecture (CDA). Int J Med Inform. 2005; 74(2-4): 245-56. PMID: 15694631 DOI: 10.1016/j.ijmedinf.2004.09.005 [PubMed]

21. Heitmann KU, Schweiger R, Dudeck J. Discharge and referral data exchange using global standards--the SCIPHOX project in Germany. Int J Med Inform. 2003; 70(2-3): 195-203. PMID: 12909170 DOI: 10.1016/s1386-5056(03)00036-4 [PubMed]

22. Channin DS. Integrating the healthcare enterprise: A primer. Part 2. Seven brides for seven brothers: The IHE integration profiles. Radiographics. 2001;21(5):1343-50.

23. Siegel EL, Channin DS. Integrating the Healthcare Enterprise: a primer. Part 1 . Introduction. Radiographics. 2001; 21(5): 1339-41. PMID: 11553842 DOI: 10.1148/radiographics.21.5.g01se391343 [ubMed]

24. Dvorak CD, Seow K, Bormann D, Larsen S, Michalski C, Ma A, et al. System and method for integration of health care records. Patent No. 20020120472. 2002.

25. Samy GN, Ahmad R, Ismail Z. Security threats categories in healthcare information systems. Health Informatics J. 2010; 16(3): 201-9. PMID: 20889850 DOI: $10.1177 / 1460458210377468$ [PubMed]

26. Barber B, Garwood D, Skerman P. Security in hospital information systems. International Journal of Biomedical Computing. 1995; 39(1): 133-8.

27. Sharifian R, Nematollahi M, Monem H, Ebrahimi F. Evaluating the security safeguards in hospital information system according to the health insurance portability and accountability act of university hospitals in Shiraz university of medical sciences, Iran. Health Information Management. 2013; 10(1): 35-46

28. Viangteeravat T, Anyanwu MN, Nagisetty VR, Kuscu E, Sakauye ME, Wu D. Clinical data integration of distributed data sources using health level seven (HL7) v3-RIM mapping. J Clin Bioinforma. 2011; 1: 110. PMID: 22104558 DOI: $10.1186 / 2043-9113-1-32$ [PubMed]

29. Bowman S. Coordinating SNOMED-CT and ICD-10. J AHIMA. 2005; 76(7): 60-1. PMID: 16097126 [PubMed] 